\title{
Causes for antiretroviral regimen change among HIV/AIDS patients in Addis Ababa, Ethiopia
}

\author{
YOHANNES T. JIMA ${ }^{1 *}$, MULUGETA T. ANGAMO ${ }^{2}$ and NASIR-TAJURE WABE ${ }^{2}$ \\ ${ }^{1}$ Amhara Regional Health Bureau, Bahir Dar, Ethiopia \\ ${ }^{2}$ Clinical Pharmacy course team, Department of Pharmacy, College of Public Health and Medical Sciences, \\ Jimma University, Jimma, Ethiopia
}

\begin{abstract}
Highly active antiretroviral therapy has markedly decreased the morbidity and mortality due to HIV/AIDS. Once antiretroviral therapy (ART) is initiated, patients generally remain on medications indefinitely. A switch in the antiretroviral regimen is often necessary because of both acute and chronic toxicities, concomitant clinical conditions, and development of virologic failure. The objective of this study was to assess the causes of initial highly active antiretroviral therapeutic regimen changes among patients on ART in Addis Ababa, Ethiopia. This was a retrospective cross-sectional study conducted from January 1 to March 1, 2010 in two primary hospitals and one health centre in central Ethiopia. Information cards of HIV/AIDS patients who have had their antiretroviral regimen switched were reviewed. Data from patients below 18 years old and those who did not switch HAART regimen were excluded. Data were then analyzed using SPSS for windows version 16.0. A total of 300 patients' information card was reviewed and the mean age of the patients was $38.6 \pm 7$ years. Females accounted for $59 \%$ (177) of the total patients. The most common first regimen before first switch was D4T/3TC/NVP (63\%) and D4T/3TC/EFV (18\%). The main reasons for modification were toxicity (65\%), comorbidity (25\%), pregnancy (5\%) and treatment failure (3\%). The main types of toxicities observed were peripheral neuropathy (39\%), rash (20\%) and anaemia (13.33\%). Drug toxicity was the main reason for modification of initial antiretroviral regimen and initial Efavirenz-based regimens are less likely to be changed. The occurrence of drug toxicity should be assessed early among patients commencing HAART and health professionals should be empowered to make appropriate regimen changes.
\end{abstract}

Keywords: HIV, antiretroviral therapy changes, drug toxicity, Ethiopia

\section{Introduction}

Highly active antiretroviral therapy (HAART) has revolutionized the treatment of HIV and has transformed this infection from a fatal to a medically managed disease. However, HAART regimens are complex, and non-adherence to medication is common. HAART regimens commonly require changes, which often involve switches of multiple medications simultaneously. However, no investigation has examined the effect of antiretroviral regimen changes on medication adherence (Haubrich et al., 1999; Golin et al., 1999; Paterson et al., 2000).

According to the standard treatment guidelines of Ethiopia; criteria for initiating ART for adults and adolescents include: if CD4 testing available; WHO stage IV diseases irrespective of CD4 cell count and WHO stage I, II or III with CD4 counts below $200 / \mathrm{mm}^{3}$. If CD4 testing unavailable; WHO stage II or III disease with a total lymphocyte count below $1200 / \mathrm{mm}^{3}$ (FMoH, 2007).

Once antiretroviral therapy (ART) is initiated, patients generally remain on medications indefinitely. A switch in the antiretroviral (ARV) regimen is often necessary because of both acute and chronic toxicities, concomitant clinical conditions, and

\footnotetext{
*Correspondence: Yohannes T. Jima; E-mail: yohannestabor@yahoo.com
} 
development of virologic failure. The approach to patients who need to switch ART will differ depending on several issues, including the reason for change, the amount of previous ART experience, and the available treatment options. For example, when patients develop an adverse effect to a drug during their first ARV regimen, effective treatment may be easily accomplished by substituting another agent for the offending drug in the regimen. At the opposite end of the spectrum are patients with advanced HIV disease, who have experienced toxicities, virologic failure, and drug resistance during multiple past treatment regimens and thus require a new treatment regimen (Timothy et al., 2006; FMoH, 2007). Rationale for treatment switch may be either pre-emptive (because of the risk of long term toxicity, poor adherence, a desire for pregnancy, a sub-optimal regimen, co-morbidity), when virological suppression is usually retained or reactive to virological rebound or (because of resistance or poor adherence) or an established acute and/or chronic toxicity (Palella et al., 1998; Timothy et al., 2006; Hart, 2007).

With the scaling up access to ART in Ethiopia, there is an opportunity to better understand the benefits and drawbacks of these regimens. Data on modification of highly active antiretroviral therapy are scarce among HIV patients in Ethiopia, so this data can potentially provide a long term strategic approach to initial and subsequent decisions regarding ART. The objective of this study was, therefore, to assess the reasons for changing initial highly active antiretroviral therapeutic regimen among patients on ART in Addis Ababa.

\section{Materials and Methods}

\section{Study design and population}

A retrospective cross sectional study design of HIV patients who had ART regimen changes at two primary hospitals (St Paulo's Hospital, Ethio-Tebib Hospital) and one health centre (Addis Ketema) in Addis Ababa, Ethiopia was used. The study was conducted from January 1 to March 1, 2010. The sample size was determined taking the following assumptions: since there was no previous study in the area, the estimated prevalence rate of population whose ARV regimen changed due to different reason was taken as 50\%, with confidence of $95 \%$, margin of error $5 \%$ and non-response rate of $5 \%$. Accordingly the sample size, therefore, was equivalent to 403 . However, the source population was less than 10,000 , thus, with the population adjustment formula; the sample size was reduced to 359. The study population consisted of patient information cards of HIV/AIDS patients who have been switched antiretroviral therapy regimen with the exclusion of HIV infected patient below 18 years of age and patients who did not switch HAART regimen.

\section{Data collection}

Before the start of the actual data collection, the data collection format and the whole method was pre-tested on randomly selected patients' clinical information records to ensure their completeness. Possible corrections were made on the data collection format through in-depth discussion with experienced pharmacists and internists working in College of Public Health and Medical Science of Jimma University. Data collection format contains sociodemographic variables and patient information, and clinical information and antiretroviral treatment information such as CD4 count on the start (CD4 cell count/NL), WHO stage at the start of treatment, initial (starting) regimen, date on treatment started, date initial ARV drug regimen switch made, duration on initial ARV therapy before fist switch, regimen switched to, reason for changing regimen and type of treatment failure were included. 


\section{Data analysis}

Data was then checked, entered, coded and analyzed using SPSS for windows version 16.0 statistical software package. For descriptive statistics, results were expressed in terms of percentages in tables. Categorical variables were compared using chi-square test. Statistical significance was considered at $\mathrm{p}$-value $<0.05$.

\section{Ethical considerations}

The study was approved by the Ethics Committee of Jimma University. Official letter was written to the three health institutions to secure permission. The confidentiality of the data obtained was assured and no disclosure of any name of the patients, the health care provider or drug product in relation to the finding was made.

\section{Results}

A total of 300 patient information cards (with a response rate of $84 \%$ ) whose initial antiretroviral treatment regimen changed in St. Paulo's Hospital, Ethio-Tebib Hospital and Addis Ketema Health Centre were reviewed. The mean age (+SD) of patients was 38.6 \pm 7 years. Majority, 234 (78\%) were between the ages of $18-49$ years. Females accounted for the largest proportion (59\%).

Table 1: General review results of the participants' information sheet and physician diagnosis card in Addis Ababa, January 2010

\begin{tabular}{lll}
\hline Variable & Response & Frequency (\%) \\
\hline Initial CD4 cells count/mm $\mathrm{mm}^{3}$ & $<50$ & $44(15)$ \\
& $51-100$ & $71(24)$ \\
& $101-200$ & $123(41)$ \\
Initial HAART regimen & $>200$ & $48(16)$ \\
& Information mission & $14(5)$ \\
& D4T/3TC/NVP & $189(63)$ \\
& D4T/3TC/EFV & $53(18)$ \\
Common reasons for modification & AZT/3TC/NVP & $39(13)$ \\
& AZT/3TC/EFV & $19(6)$ \\
& Toxicity & $195(65)$ \\
& Co-morbidity & $75(25)$ \\
& Pregnancy & $15(5)$ \\
Toxicities reported as a reason for treatment change & Treatment failure & $9(3)$ \\
& Adherence difficulty & $6(2)$ \\
& Peripheral neuropathy & $77(39)$ \\
& Rash & $39(20)$ \\
& Anaemia & $26(13)$ \\
& CNS manifestation & $22(11)$ \\
& Lipidostrophy & $21(11)$ \\
& Others* & $10(5)$ \\
\hline
\end{tabular}

* Hepatoxicity, GIT symptoms

From a total of 300 patents, $123(41 \%), 71$ (24\%) and 48 (16\%) had initial CD4 count of 101-200 cells $/ \mathrm{mm}^{3}$, 51-100 cells $/ \mathrm{mm}^{3}$ and $>200$ cells $/ \mathrm{mm}^{3}$, respectively. Regarding to the initial regimen, 189 (63\%) patients were on D4T/3TC/NVP, 53 (18\%) were on D4T/3TC/EFV, 39 (13\%) were on $\mathrm{AZT} / 3 \mathrm{TC} / \mathrm{NVP}$ and $19(6 \%)$ were on $\mathrm{AZT} / 3 \mathrm{TC} / \mathrm{EFV}$. The common reasons reported for modification of initial regimens were toxicity 195 (65\%), co-morbidity 75 (25\%) and planning 
pregnancy or being pregnant $15(5 \%)$, treatment failure $9(3 \%)$ and adherence difficulty $6(2 \%)$. From all toxicities 195 (65\%) reported, peripheral neuropathy accounting 77 (39\%) was the most commonly followed by rash 39 (20\%), anaemia 26 (13\%) and central nervous system manifestations 22 (11\%) (Table 1).

From all toxicities, majority 110 (56\%) was due to D4T/3TC/NVP regimen followed by 42 (22\%) with D4T/3TC/EFV and 27 (14\%) with AZT/3TC/NVP. Initial regimen change due to comorbidity 72 (37\%) was with $\mathrm{D} 4 \mathrm{~T} / 3 \mathrm{TC} / \mathrm{NVP}$, treatment failure 4 (2\%) with $\mathrm{D} 4 \mathrm{~T} / 3 \mathrm{TC} / \mathrm{NVP}$, planning pregnancy 13 (7\%) with $\mathrm{D} 4 \mathrm{~T} / 3 \mathrm{TC} / \mathrm{EFV}$ and adherence difficulty 4 (2\%) with D4T/3TC/NVP (Table 2).

Table 2: Common reasons for modification of initial regimen with respect to specific initial regimen

\begin{tabular}{llllll}
\hline Reason & \multicolumn{5}{c}{ Regimen, N (\%) } \\
& D4T/3TC/NVP & D4T/3TC/EFV & AZT/3TC/NVP & AZT/3TC/EFV & Total \\
\hline Toxicity & $110(37)$ & $42(14)$ & $27(9)$ & $16(5)$ & $195(65)$ \\
Co morbidity & $72(24)$ & 0 & $3(1)$ & 0 & $75(25)$ \\
Pregnancy & 0 & $13(4)$ & 0 & $2(1)$ & $15(5)$ \\
Treatment failure & $4(1)$ & $1(0.3)$ & $4(1)$ & 0 & $9(3)$ \\
Adherence difficulty & $4(1)$ & 0 & $2(1)$ & 0 & $6(2)$ \\
Total & $190(63)$ & $56(19)$ & $36(12)$ & $18(6)$ & $300(100)$ \\
\hline
\end{tabular}

Peripheral neuropathy was due to D4T containing regimens of D4T/3TC/NVP 62 (32\%) and D4T/3TC/EFV 15 (8\%) whereas rash was due to NVP containing regimens of D4T/3TC/NVP 34 (17\%) patients and $\mathrm{AZT} / 3 \mathrm{TC} / \mathrm{NVP} 5$ (3\%). Anaemia was reported due to AZT containing regimens of $A Z T / 3 T C / N V P 14$ (7\%) and $A Z T / 3 T C / E F V 12$ (6\%) whereas CNS toxicities reported as dizziness, nightmare, and sleep disturbance were due to EFV contains regimens of D4T/3TC/NVP 20 (10\%) and AZT/3TC/EFV 2 (1\%). Lipodystrophy was due to D4T containing regimens of $\mathrm{D}_{4} \mathrm{~T} / 3 \mathrm{TC} / \mathrm{NVP} 19$ (10\%) and D4T/3TC/EFV 2 (1\%). The other toxicities observed were nausea, vomiting and hepatoxicity which were mainly due to NVP and AZT containing regimens of $\mathrm{D}_{4} \mathrm{~T} / 3 \mathrm{TC} / \mathrm{NVP} 4$ (2\%) and $\mathrm{AZT} / 3 \mathrm{TC} / \mathrm{NVP} 3$ (2\%), respectively (Table 3 ).

Table 3: Toxicities reported as a reason for initial treatment regimen change in Addis Ababa

\begin{tabular}{lllllll}
\hline Toxicities & \multicolumn{7}{c}{ Initial treatment regimen, $\mathbf{N}(\%)$} & P-value \\
& D4T/3TC/NVP & D4T/3TC/EFV & AZT/3TC/NVP & AZT/3TC/EFV & Total & \\
\hline Peripheral & $62(32)$ & $15(8)$ & 0 & 0 & $77(39)$ & \\
neuropathy & & & & & & \\
Rash & $34(17)$ & 0 & $5(3)$ & 0 & $39(20)$ & \\
Anaemia & 0 & 0 & $14(7)$ & $12(6)$ & $26(13)$ & \\
CNS toxicity & 0 & $20(10)$ & 0 & $2(1)$ & $22(11)$ & \\
Lip atrophy & $19(10)$ & $2(1)$ & 0 & 0 & $21(11)$ & \\
Other* & $4(2)$ & $1(1)$ & $2(1)$ & $3(2)$ & $10(5)$ & \\
Total & $119(61)$ & $38(19)$ & $21(11)$ & $17(9)$ & $195(100)$ & 0.000 \\
\hline
\end{tabular}

* Hepatoxicity, GIT symptoms

From a total of 300 patients, 112 (37\%) stayed on initial regimen for only 12 weeks followed by 79 (26\%) for 13 to 24 weeks, 67 (22\%) for 25 to 48 weeks, 35 (12\%) for 47 to 72 weeks and 7 (2\%) for more than 72 weeks, otherwise they were switched to another regimen (second line). Similarly, 190 (63\%) patients stayed on D4T/3TC/NVP regimen followed by 53 (18\%) on D4T/3TC/EFV, 36 (12\%) on AZT/3TC/NVP and 21 (7\%) on AZT/3TC/EFV (Table 4). 
Table 4: Weeks of stay on initial antiretroviral treatment versus initial treatment regimen

\begin{tabular}{lllllll}
\hline Initial regimen & \multicolumn{6}{l}{ Weeks of stay on initial therapy, $\mathbf{N}(\%)$} \\
\hline & Start-12 weeks & $13-24$ & $25-48$ & $49-72$ & $>72$ & Total \\
D4T/3TC/NVP & $73(24)$ & $51(17)$ & $37(12)$ & $25(8)$ & $4(1)$ & $190(63)$ \\
D4T/3TC/EFV & $17(6)$ & $13(4)$ & $15(5)$ & $7(2)$ & $1(0.3)$ & $53(18)$ \\
AZT/3TC/NVP & $18(6)$ & $9(3)$ & $6(2)$ & $2(1)$ & $1(0.3)$ & $36(12)$ \\
AZT/3TC/EFZ & $4(1)$ & $6(2)$ & $9(3)$ & $1(0.3)$ & $1(0.3)$ & $21(7)$ \\
Total & $112(37)$ & $79(26)$ & $67(22)$ & $35(12)$ & $7(2)$ & $300(100)$ \\
\hline
\end{tabular}

From a total of 195 (65\%) patients whose initial regimen modification made due to toxicities, $61(31 \%)$ were in the first 12 weeks followed by $52(27 \%)$ in the $24-48$ weeks period. Similarly, from a total of 75 (25\%) patients whose initial regimen change was due to co-morbidity, 46 (24\%) were in the first 12 weeks period (Table 5).

Table 5: Weeks of stay on initial antiretroviral treatment versus reasons for changing the regimen

\begin{tabular}{lllllll}
\hline $\begin{array}{l}\text { Reasons for } \\
\text { change }\end{array}$ & \multicolumn{7}{l}{ Weeks of stay on initial therapy, $\mathbf{N}(\%)$} \\
& Start-12 weeks & $\begin{array}{l}13-24 \\
\text { weeks }\end{array}$ & $25-48$ weeks & $49-72$ weeks & $\begin{array}{l}\text { >72 } \\
\text { weeks }\end{array}$ & Total \\
Toxicity & $61(31)$ & $49(25)$ & $52(27)$ & $30(15)$ & $3(2)$ & $195(65)$ \\
Co-morbidity & $46(24)$ & $22(11)$ & $6(3)$. & $1(0.5)$ & 0 & $75(25)$ \\
pregnancy & $3(2)$ & $5(3)$ & $4(2)$ & $2(1)$ & $1(0.5)$ & $15(5)$ \\
Treatment failure & 0 & 0 & $4(2)$ & $2(1)$ & $3(2)$ & $9(3)$ \\
$\begin{array}{l}\text { Adherence } \\
\text { difficulty }\end{array}$ & $2(1)$ & $3(2)$ & $1(0.5)$ & 0 & 0 & $6(2)$ \\
Total & $112(37)$ & $79(26)$ & $67(22)$ & $35(12)$ & $7(2)$ & $300(100)$ \\
\hline
\end{tabular}

\section{Discussion}

Rationale for treatment switch could be due to risk of toxicity, poor adherence, a desire for pregnancy, treatment failure or co morbidity (Ammassari, 2001). Majority of the patients were on D4T based regime of D4T/3TC/NVP (63\%). This study is consistent with the research done in southern India where D4T/3TC/NVP accounted for 63\% (Kumara \& Vallabhaneni, 2006) and with that in Asia where D4T/3TC/NVP accounted for $37 \%$. However, our findings are not in agreement with those reported by Hart (2007), where AZT/3TC/EFV (44\%) regimen accounted for the majority of the patients' initial HAART regimen. The probable reason is the difference in patient conditions, co-morbid situation or contraindications.

Like several other studies (D'Arminio et al., 2000; Dorrucci et al., 2001; Mocroft et al., 2001; O'Brien et al., 2003; Kumara \& Vallabhaneni, 2006; Kiguba, 2007; Hart, 2007; Cesar et al., 2010), the most known causes of ARV switching, in the present study, was toxicity with significant heterogeneity in the distribution of adverse events, which is also statistically significant. The patients were with more advanced disease at baseline, which may necessitates higher rates of regimen change/discontinuation due to adverse events.

Unlike in a study in Peru (Bangsberg et al., 2006) peripheral neuropathy was the most common reason for modification in our study. This is most probably due to the reason that most of the patients in this study were on D4T-based regimen of D4T/3TC/NVP and D4T/3TC/EFV and were initially with advanced HIV infection. Study done in Peru reported anaemia as a main reason for discontinuation, and associated this finding with the use of standard $600 \mathrm{mg}$ ZDV in low weight patients ((Bangsberg et al., 2006). In the current study, 
the distribution of change in regimen due to skin rash and anaemia were the second and third most common reasons for modification, respectively. Rash was mainly due to NVP containing regimen $\mathrm{D} 4 \mathrm{~T} / 3 \mathrm{TC} / \mathrm{NVP}$ and $\mathrm{AZT} / 3 \mathrm{TC} / \mathrm{NVP}$. Anaemia was due to AZT containing regimen AZT/3TC/NVP and AZT/3TC/EFV. Unlike the Peru's study (Bangsberg et al., 2006), where the low rates of HAART change was due to anaemia, in this study, was likely to be due to lack of adequate baseline anaemia assessment and the fact that no close monitoring of anaemia at the study site.

Co-morbidities in patients with advanced disease and concurrent treatments for opportunistic diseases may affect antiretroviral tolerance and thereby increase risk of toxicities (Cesar et al., 2010). Co-morbidity was the other cause for HAART switch. Tuberculosis was the only co-morbidity disease reported in this study. This is consistent with the study in the United Kingdom (Hammer, 2020) and Cote d'lvoire (Palombi et al., 2008). The probable suggestion for this NVP switch to EFV is the overlapping drug toxicity effect of NVP with anti-TB drugs which is hepatotoxicity, and the potential for drug interaction since NVP is CYP $3 \mathrm{~A} 4$ enzyme inducer.

Efavirenz-based regimens had the lowest hazard for change relatively. However, the increased hazard for change of efavirenz-based regimens was pronounced during pregnancy or when planning pregnancy. Planning pregnancy or being pregnant was the third major reason for modifying antiretroviral drugs in this study. Similar findings have been reported in other studies elsewhere (Hart, 2001; Cesar, 2010; Bangsberg et al., 2006; Mess et al., 2010). This switch was mainly due to the teratogenic effect of EFV which should mainly be avoided during the first trimester pregnancy.

As expected and previously reported (Kumara \& Vallabhaneni, 2006; D’Arminio et al., 2000; Keiser et al., 2008; Palombi et al., 2008; Zhon et al., 2010), patients were more likely to change therapy shortly after HAART initiation because of adverse events rather than treatment failure. Treatment failure was given as the reason for change in 9 (3\%) of patients in current study. This occurred mainly due to D4T containing regimen of D4T/3TC/NVP 4(1\%) and $\mathrm{AZT} / 3 \mathrm{TC} / \mathrm{NVP} 4(1 \%)$ of the patients. This may be explained by differences in primary resistance to NRTIs or NNRTIs containing regimens (Dorrucci et al., 2001). However, some studies have reported higher treatment failure as a reason of regimen switch ((Kumara \& Vallabhaneni, 2006; Hart, 2007; Kiguba, 2007; Mess et al., 2010). In the study in Cote d'lvoire, treatment failure was observed in $12 \%$ patient (Keiser et al., 2008) and according to the study in India, treatment failure accounts for $14 \%$ of the reasons for modifying therapy (Zhon et al., 2010). According to a study in Uganda (Kiguba, 2007), immunologic failure alone predicated virologic failure in $56 \%$ of patients. This may be due to lack of viral load measuring device and lack of continuous monitoring of patients CD4 count and on occurrence of opportunistic infection in the study setting of this study.

Cost was one of the major reasons for discontinuation and modification of ARV drugs according to the study conducted in India (Zhon et al., 2010) and Uganda (Kiguba, 2007). But it was not a reason for modification of ARV regimen in our current study due to cost free (fee-free) provision of ARV drugs for the patient in Ethiopia.

A statistically significant association was observed between common reasons for modifications of regimen and initial regimen. Similarly, toxicities like peripheral neuropathy, rash and anaemia were significantly associated with respective initial regimen. However, weeks of stay on initial regimen had no significant association with initial regimen rather weeks of stay on initial regimen had significant statistical association with respect to common reasons for regimen changes because the longer stay the higher probability of 
regimen change. This was consistent with the findings reported in the United Kingdom (Hammer et al., 2002), India (Kumara \& Vallabhaneni, 2006) and Uganda (Kiguba, 2007).

The findings of this study should be interpreted with some limitations. These include, lack of appropriately filled patient information sheet, and the retrospective study might not allow for a direct investigation of causal relationship between the factors studied and the outcome of interest. The study collected the main reasons as reported by physician for modification of treatment, but reasons for modification are often interrelated.

In conclusion, the result of this study indicated toxicity as the main reason for modification of initial ARV drugs among the study population. Peripheral neuropathy was the leading cause for modification of HAART. Efavirenz-based regimens had the lowest hazard for change relatively though, responsible for switched in pregnancy related cases. Tuberculosis was the only co-morbidity disease reported in this study and all patients who switched were due to occurrence of tuberculosis after starting ARV drugs on NVP based regimen. Adherence difficulty was the least reasons for ARV regimen change. There should be a viral load measuring device in each of the health institution to establish the extent of treatment failure. As much as possible, clinicians should stick to the national antiretroviral drug use guidelines for the management and follow up of patients receiving HAART. The national policy makers should up-date the guidelines as soon as possible. Switching should be based on a risk benefit ratios, especially for pregnant HIV patients. The factors associated with modification of HAART observed in this study should be investigated further in longitudinal studies of ART utilization.

\section{References}

Ammassari, A. (2001) Self-reported symptoms and medication side effects influence adherence to HAART in persons with HIV infections. Journal of Acquired Immune Deficiency Syndromes 28, 445-449.

Bangsberg, D.R., Acosta, E.P., Gupta, R., Guzman, D., Riley, E.D, Harrigan, P.R., Parkin, N. \& Deeks, S.G. (2006) Adherence-resistance relationships for protease and nonnucleoside reverse transcriptase inhibitors explained by virological fitness. AIDS 20, 223-231.

Cesar, C.M., Shepherd, B.E., Krolewiecki, A.J., Fink, V.I., Schechter M., Tuboi, S.H., Wolff M., Pape, J.W., Leger P., Padgett D., Madero, J.S., Gotuzzo, E. \& Sued, O. (2010) Rates and Reasons for Early Change of First HAART in HIV-1-Infected Patients in 7 Sites throughout the Caribbean and Latin America. PLOS ONE 5 (6), 1-10

D'Arminio Monforte, A., Lepri, A. C., Rezza, G., Pezzotti, P., Antinori, A., Phillips, A. N., Angarano, G., Colangeli, V., Luca, A. De, Ippolito, G., Caggese, L., Soscia, F., Filice, G., Gritti, F., Narciso, P., Tirelli, U. \& Moroni, M.(2000) Insights into the reasons for discontinuation of the first highly active antiretroviral therapy (HAART) regimen in a cohort of antiretroviral naïve patients. Italian Cohort of Antiretroviral-Naïve Patients. AIDS 14, 499-507.

Dorrucci, M.A., Pezzotti, P.A., Grisorio, B.B., Minardi, C.C., Muro, M.S.D., Vullo, V.E., D'Arminio, M.A. (2001) Time to discontinuation of the first highly active antiretroviral therapy regimen: a comparison between protease inhibitor- and non-nucleoside reverse transcriptase inhibitor-containing regimens. AIDS 15, 1733-1736.

$\mathrm{FMoH}$ (2007) Guidelines for Implementation of the Antiretroviral Therapy Program in Ethiopia, $1^{\text {st }}$ ed, 2007, 2:11-3. Ethiopian Federal Ministry of Health. 
Golin, C., Liu, H., Hays, R., Ickovics, J., Beck, K., Miller, L., Kaplan, A. \& Wenger, N. (1999) Patient factors associated with and self-reported reasons for non-adherence to antretroviral therapy. Presented at the $22^{\text {nd }}$ annual meeting of the Society of General Internal Medicine. Journal of General Internal Medicine 14 (Suppl 2), 35.

Hammer, S.M. (2002) Increasing choice for HIV Therapy. New England Journal of Medicine 346, 2022-2023.

Hart, E. (2007) National review of first treatment change after starting highly active antiretroviral therapy in antiretroviral naïve patients. HIV Medicine 8, 18-191.

Haubrich, R.H., Little, S.J., Currier, J.S., Forthal, D.N., Kemper, C.A., Beall, G.N., Johnson D., Dubé, M.P., Hwang, J.Y. \& McCutchan, J.A. (1999) The value of patient reported adherence to antiretroviral therapy in predicting virologic and immunologic response. California Collaborative Treatment Group. AIDS 13, 1099-1107.

Kagay, C.R., Porco, T.C., Liechty, C.A., Charlebois, E., Clark, R., Guzman, D., Moss, A.R., Bangsberg, D.R. (2004) Modeling the impact of modified directly observed antiretroviral therapy on HIV suppression and resistance, disease progression, and death. Clinical Infectious Disease 38 (Suppl 5), S414-420.

Keiser, O., Orrell, C., Egger, M., Wood, R., Brinkhof, M.W.G, Furrer, H., Cutsem, G.V.,Ledergerber B. \& Boulle A. (2008) Public-health and individual approaches to antiretroviral therapy: Township South Africa and Switzerland compared. PLoS Medicine 5 (7), e148.

Kiguba, M.N. (2007) Discontinuation and modification of highly active antiretroviral therapy in HIV-infected Ugandans: Prevalence and Associated factors. Journal of Acquired Immune Deficiency Syndromes 45, 218-223.

Kumara, S.N. \& Vallabhaneni, S. (2006) Reasons for modification of generic highly active antiretroviral therapeutic regimens among patients in southern India. Journal of Acquired Immune Deficiency Syndromes 41, 53-8.

Messou, E., Anglaret, X., Duvignac, J., Konan-N'dri, E., Komena, E., Gnokoro, J., Karcher, S., Tanoh, A., N'dri-Yoman, T. \& Seyler, C. (2010) Antiretroviral treatment changes in adults from Cote d'lvoire: the role of tuberculosis and pregnancy. AIDS 24, 93-99.

Mocroft, A., Youle M., Moore, A., Sabin, CA., Madge, S., Lepri, A.C., Tyrer, M., Chaloner, C., Wilson, D., Loveday, C., Johnson, M.A. \& Phillips, A.N. (2001) Reasons for modification and discontinuation of antiretrovirals: results from a single treatment centre. AIDS 15, 185-194.

O’Brien, M.E., Clark, R.A., Besch, C.L., Myers, L. \& Kissinger, P. (2003) Patterns and correlates of discontinuation of the initial HAART regimen in an urban outpatient cohort. Journal of Acquired Immune Deficiency Syndromes 34, 407-414.

Palella, F.J., Palella, F.J. Jr., Delaney, K.M., Moorman, A.C., Loveless, M.O., Fuhrer J., Satten, G.A., Aschman, D.J., SD H. (1998) Declining morbidity and mortality among patients with advanced human immunodeficiency virus infection. New England Journal of Medicine 338, 853-860.

Palombi, L., Marazzi, M. C., Guidotti, G., Germano, P., Buonomo, E., Scarcella, P.,Doro, A.A. , Zimba Ines Da, V.M. , Magnano, M., Lio S., De Luca, A. (2009). Incidence and Predictors of Death, Retention, and Switch to Second-Line Regimens in AntiretroviralTreated Patients in Sub-Saharan African Sites with Comprehensive Monitoring Availability. Clinical Infectious Disease 48, 115-122.

Park, W.B., Choe, P.G., Kim, S.H., Jo, J.H., Bang, J.H., Kim, H.B., Kim, N.J., Oh, M.D. \& Choe, K.W. (2007) Early modification of initial HAART regimen. Retroviruses 23, 798-800. 
Paterson, D.L., Swindells, S., Mohr, J., Brester, M., Vergis, E.N. \& Squier, C. (2000) Adherence to protease inhibitor therapy and outcomes in patients with HIV infection. Annals Internal Medicine 133, 21-30.

Timothy, W., Marshall, G. \& Roy, M. (2006) HIV insight knowledge base chapter changing Antiretroviral Therapy: why, when and how. Journal of Acquired Immune Deficiency Syndromes 12, 782-789.

Zhou, J., Li, P.C., Kumarasamy, N., Boyd, M., Chen, Y.M., Sirisanthana, T., Sungkanuparph, S. \& Oka, S. (2010) Deferred medication of antiretroviral regimen following documented treatment failure in Asia: results from the Treat Asia HIV Observational Database. HIV Medicine 11, 31-39. 